\title{
Dynamic nonlinear feedback for temperature control of continuous stirred reactor with complex behavior
}

\author{
Pablo A. López Pérez ${ }^{1}$, Ricardo Aguilar-López ${ }^{2 *}$ \\ 1,2 Departamento de Biotecnología y Bioingeniería \\ Centro de Investigación y de Estudios Avanzados del I.P.N. \\ CINVESTAV-IPNAv. Instituto Politécnico Nacional No. 2508, San Pedro Zacatenco, \\ México, D.F. 07360 \\ *raguilar@cinvestav.mx
}

\begin{abstract}
The main objective of this work is to present an alternative methodology for the design of a class of integral high order slidingmode controller applied to a class of continuous chemical reactor with complex behavior for temperature tracking purposes. The proposed design is based on the differential geometry framework, where the named reaching trajectory contains a high order sliding mode term in order to diminish chattering. Considering that the proposed technique is model based, an observerbased uncertainty estimator is coupled, which provides robustness against model uncertainties and noisy measurements. Numerical simulations are performed in order to show the capacities of the proposed controller, which is compared with other nonlinear methodologies.
\end{abstract}

Keywords: Chemical Reactor, Temperature tracking, observer based high order sliding mode controller, robust performance.

\section{RESUMEN}

El principal objetivo de este trabajo es presentar una metodología alternativa para el diseño de una clase de controlador integral de tipo modo deslizante de alto orden, que es aplicado a un reactor químico continuo con comportamiento complejo con fines de seguimiento de temperatura. El diseño propuesto se realiza bajo el marco de la geometría diferencial, donde la denominada trayectoria guía contiene un termino de tipo deslizante de alto orden con el fin de disminuir los problemas de oscilaciones. Considerando que la metodología propuesta está basada en el modelo de la planta, se acopla un estimador de incertidumbres basado en un observador, el cual provee condiciones de robustez contra errores de modelado y ruido en las mediciones. Simulaciones numéricas son realizadas para mostrar las capacidades del controlador propuesto, el cual es comparado con otras metodologías no lineales.

Palabras clave: Reactor químico, seguimiento de temperatura, controlador de modo deslizante de alto orden basado en observador, desempeño robusto.

\section{Introduction}

The continuous stirred tank reactor (CSTR) is a workhorse at many chemical plants, frequently serving multi-purpose production objectives for fine and specialty chemicals. It can handle not only reaction, also solvent extraction, crystallization and distillation. The successful installation of such a reactor depends, to a great degree, upon the proper design of its temperature-control system.

For reacting systems, the endothermic reactions exhibit a marked degree of self-regulation in regard to thermal stability and do not need further consideration. In contrast, exothermal reactions require detailed understanding of kinetics and thermodynamics, in order to obtain the rate of heat generation. Meanwhile, heat removal capability, as a function of the resistances to heat transfer, temperature difference and transfer area, becomes very important to manage exothermal reactions (Froment and Bischoff, 1990; Ogunnaike and Ray, 1994). A chemical reaction that goes out of control and runs away can create serious incidents, risking people, environment and equipment. Reaction temperature increases lead to a higher rate of 
reaction and pose a risk of thermal runaway if heat cannot be removed fast enough; any reduction in heat-transfer area due to a decrease in reactor contents adds to the problem (Aguilar R. et al. 1997). Sometimes cooling devices are not able to provide stability; however, removing heat by boiling one or more components may provoke stability due to the property of constant temperature at boiling point (Aris and Admundson, 1958). An important fact that has to be considered is that the rate at which heat is removed increases linearly with reaction temperature, whereas the rate at which heat is produced increases exponentially. Once control of the reaction is lost, temperature can rise rapidly leaving little time for correction.

From the economics point of view, one of the biggest challenges facing process engineers will be the reduction of variable costs whilst maintaining product quality. Advanced process control is one of the most effective tools available to fulfill this objective, especially on established plants. As systems become more complex, another important aspect is the reliability of the implemented systems. Here, the reliability of hardware and software are issues which have to be addressed. Allied to this is the requirement for suitably designed man-machine interfaces to enable efficient and reliable information transfer and to facilitate systems management (Shinskey, 1994). With regard to the primary modules making up an advanced control project, neural networks, nonlinear systems theory, robust control, knowledge based systems are areas which appear to have captured the attention of both researchers and practitioners in the field of control engineering. This trend will continue well into the next years. Areas that will receive particular attention will be techniques that will translate raw data into useful information; improved measurement methods including inferential estimation; multivariable non-linear predictive control and formal techniques for analyzing the integrity of neural network based methodologies (Azlan Hussain, 1999).

All information is of value, and should not be discarded just because they do not conform to a particular model building procedure. Thus, new modeling methods are also required. These should provide a framework where a priori knowledge of the process could be combined with the various existing modeling techniques, leading to so called 'grey-box' models (Madar, et. al., 2005). The resulting models should also be amenable for utilization by the different modern controller designs, thus rendering controller synthesis independent of model types (Aguilar-López and Martínez-Guerra, 2008).

Process industries have an enormous base of manufacturing facilities which are still being run by unsophisticated or primitive control schemes. Competitive pressures will not allow any company in these industries to ignore the significant efficiencies possible through adopting modern process control technologies. A major obstacle to realizing the full potential of modern control techniques is the lack of exposure to advances in the field. This can be overcome by the development of portable computer based training packages. The current proliferation in multi-media computing systems is the ideal impetus for the development of such learning aids.

Appropriately designed nonlinear controllers would therefore be expected to perform better, using a variety of approaches such as mechanistic models via the use of differential geometric concepts (Isidori, 1995). The aim of the design is similar to the use of Taylor series expansion to linearise the nonlinear model prior to the application of linear model-based controller designs; however, instead of providing local linearization, contemporary nonlinear control strategies aim to provide 'Global Linearizing Control' (GCL) over a span based on states of the process. Global linearization is achieved by a pre- 
compensator, generating a linear relationship between the inputs to the pre-compensator and the output from the process.

Globally linearizing control is a relatively new development and much research is still being carried out to investigate the applicability of its methodology (Kolavennu, et al, 2000). In order to avoid the requirement of mechanistic models, it is possible to use neural networks, which are transformed into an equivalent state-space representation, and then GLC is designed based upon this state-space model (González, et. al., 1999); however, some problems such as the over parameterization of this kind of systems remains. Nonlinear system model imprecision may come from actual uncertainty about the plant (e.g., unknown plant parameters), or from the purposeful choice of a simplified representation of the system's dynamics. Modeling inaccuracies can be classified into two major kinds: structured (or parametric) uncertainties and unstructured uncertainties (or unmodeled dynamics). The first kind corresponds to inaccuracies on the terms actually included in the model, while the second kind corresponds to inaccuracies on the system order.

Modeling inaccuracies can have strong adverse effects on nonlinear control systems. One of the most important approaches to dealing with model uncertainty is robust control. The sliding mode control is an important robust control approach. For the class of systems to which it applies, the sliding mode controller design provides a systematic approach to the problem of maintaining stability and consistent performance in the face of modeling imprecision (Levant, 2001). On the other hand, by allowing the tradeoffs between modeling and performance to be quantified in a simple fashion, it can illuminate the whole design process.

In control theory, the sliding mode control is a type of variable structure control where the dynamics of a nonlinear system is altered via application of a high-frequency switching control. This is a state feedback control scheme where the feedback is not a continuous function of time.

The principle of the sliding mode control is to forcibly constrain the system, by suitable control strategy, to stay on the sliding surface on which the system will exhibit desirable features. When the system is constrained by the sliding control to stay on the sliding surface, the system dynamics are governed by reduced order system (Edwards and Spurgeon, 1998, Utkin, et al, 1999).

However, as it is well known, this switching happens at any instant the state trajectories cross the switching hyper-plane, this leads to the named chattering phenomenon caused by the discontinuity of the input injection, which is undesirable in most applications. To avoid the above drawback, a kind of sliding-mode techniques, named high order sliding-mode, has been developed; several papers with application to control and observer design have been presented in the open literature (Camacho and Smith, 2000; Levant, 1993 and 2001, Bowong, et. al. 2004, Laghrouche, et al, 2007, Girin, et al, 2006),

In this work, a class of nonlinear system related with a continuous stirred tank reactor is transformed onto canonical control form via differential-geometric tools, in order to apply an observer based high-order sliding-mode framework which provides some robustness against model uncertainties and noisy measurements. The problem considered here is the classical temperature regulation task, which up to date is very important in the industrial practice.

\section{Proposed Controller}

The dynamic nonlinear model for a chemical reactor can be expressed as system (1), and the output measured for control purposes as (2) 


$$
\begin{aligned}
& \dot{x}=f(x)+g(x) u \\
& y=h(x)
\end{aligned}
$$

Here, $x \in \mathfrak{R}^{n}$ is the vector of states; $u \in \mathfrak{R}^{q}$ is the vector control input; $f(x): \mathfrak{R}^{n} \rightarrow \mathfrak{R}^{n}$ is a nonlinear, continuously differentiable and partially known vector field; $g(x): \mathfrak{R}^{n} \rightarrow \mathfrak{R}^{n}$ is an invertible matrix. Now, consider the following assumptions:

A1. For the realized control input vector $u(x(t))$, $\left(\|u(x(t))\| \leq u_{\max }\right)$, the nominal closed-loop nonlinear system (12) is, at least, quadratic stable for the controlled states; therefore, it exists a Lyapunov function $V \geq 0$ that satisfies that

$$
\frac{\partial \mathrm{V}}{\partial x}[f(x)+g(x) u] \leq-\pi_{1}\|x\|^{2}, \quad\left\|\frac{\partial \mathrm{V}}{\partial x}\right\| \leq \pi_{2}\|x\|, \quad \pi_{1}, \pi_{2}>0
$$

A2. All the trajectories $x\left(t, x_{0}\right), x_{0} \in \mathfrak{R}^{n}$ of the system (12) are bounded.

A3. The matrix field $g(x)$ is bounded, i. e. for any $x \in \mathfrak{R}^{n},\|g(x)\| \leq g^{+}<\infty$.

In accordance to the geometric-differential theoretical framework, the Lie derivative of the function $h(x)$ ) with respect to the vector field $f$, denoted as $L_{f} h(x)$, is defined as (3).

Here,

$L_{f}^{0} h(x)=h(x)$ and $L_{f}^{k+1} h(x)=L_{f}\left(L_{f}^{k} h(x)\right)$

Definition (3) implies that it exists an invertible diffeomorphism, the system is feedback linearizable, so that $(\xi, \mu)=\Psi(x)$ (Isidori, 1995); therefore, the system (1) can be expressed as

$$
\begin{aligned}
& \dot{\xi}_{i}=\xi_{i+1} ; i=1,2, \ldots, r-1 \\
& \dot{\xi}_{r}=\eta(\xi, \mu)+\varphi(\xi, \mu) u \\
& \dot{\mu}=\gamma(\xi, \mu) \\
& y=\xi_{1} \quad \text { Here, } \eta=L_{f}^{r} h(x) ; \varphi=L_{g} L_{f}^{r-1} h(x) .
\end{aligned}
$$

Sub index $r$ is defined as the relative degree of the system and it defines how many time derivatives of the measured output should be computed in order to obtain explicitly the control input.

Now consider the following change of variable [18]:

$\xi_{r+1}=\eta+\varphi u$

Therefore:

$\dot{\xi}_{r+1}=\dot{\eta}+\dot{\varphi} u+\varphi \dot{u}$

Now, the system (4) is represented as (7)

$\dot{\xi}_{i}=\xi_{i+1} ; i=1,2, \ldots, r-1$

$\dot{\xi}_{r}=\xi_{r+1}$

$\dot{\xi}_{r+1}=\dot{\eta}(\xi, \mu)+\dot{\varphi}(\xi, \mu) u+\varphi(\xi, \mu) \dot{u}$

$\dot{\mu}=\gamma(\xi, \mu)$

$y=\xi_{1}$

Here,

$\dot{\eta}=\frac{\partial \eta}{\partial \xi} \frac{\partial \xi}{\partial t}+\frac{\partial \eta}{\partial \mu} \frac{\partial \mu}{\partial t}$ and $\dot{\varphi}=\frac{\partial \varphi}{\partial \xi} \frac{\partial \xi}{\partial t}+\frac{\partial \varphi}{\partial \mu} \frac{\partial \mu}{\partial t}$.

considering the following sliding surface:

$s=\xi_{r+1}-\xi_{r+1(0)}+\int \sum_{i=1}^{r+1} a_{i} \xi_{i}=0$ 


\section{Dynamic Nonlinear Feedback for Temperature Control of Continuous Stirred Reactor with Complex Behavior, Pablo A. López Pérez, et, al, 202-217}

Note that the above stable surface provides stability to the system (7). Now, from (7) and (8), therefore, it is possible to obtain (9):

$$
\begin{aligned}
& \dot{\xi}_{i}=\xi_{i+1} ; i=1,2, \ldots, r-1 \\
& \dot{\xi}_{r}=\xi_{r+1} \\
& \dot{\xi}_{r+1}=-\sum_{i=1}^{r+1} a_{i} \xi_{i} \\
& \dot{\mu}=\gamma(\xi, \mu) \\
& y=\xi_{1}
\end{aligned}
$$

In order to compress notation, system (9) can be represented as (10)

$$
\begin{aligned}
& \dot{\xi}=A \xi \\
& \dot{\mu}=\gamma(\xi, \mu) \\
& y=\xi_{1}
\end{aligned}
$$

$$
\begin{aligned}
& \dot{\xi}_{i}=\xi_{i+1} ; i=1,2, \ldots, r-1 \\
& \dot{\xi}_{r}=\xi_{r+1} \\
& \dot{\xi}_{r+1}=\dot{\eta}(\xi, \mu)+\left(\dot{\varphi}_{o}(\xi, \mu)\right. \\
& \dot{\mu}=\gamma(\xi, \mu) \\
& y=\xi_{1} \\
& \text { or } \\
& \dot{\xi}_{i}=\xi_{i+1} ; i=1,2, \ldots, r-1 \\
& \dot{\xi}_{r}=\xi_{r+1} \\
& \dot{\xi_{r+1}}=\zeta(\xi, \mu)+\varphi_{o}(\xi, \mu) \dot{u} \\
& \dot{\mu}^{\prime}=\gamma(\xi, \mu) \\
& y=\xi_{1}
\end{aligned}
$$$$
\dot{\xi}_{r+1}=\dot{\eta}(\xi, \mu)+\left(\dot{\varphi}_{o}(\xi, \mu)+\Delta \dot{\varphi}+\dot{\varphi}_{0}\right) u+\left(\varphi_{o}(\xi, \mu)+\Delta \varphi\right) \dot{u}
$$

Here, $A=\left[\begin{array}{cccc}0 & 1 & \ldots & 0 \\ 0 & 0 & 1 & \ldots 0 \\ \ldots & \ldots & \ldots & \ldots \\ -a_{1} & -a_{2} & \ldots & -a_{r+1}\end{array}\right]$ can be considered as a Hurwitz matrix with an adequate choosing of the design parameters $a_{i}$. The $\mu \in \mathfrak{R}^{(n+1)-(r+1)}$ system is the called internal dynamics, which must be stable in order to provide detectability and stability for the system (10).

Now, suppose that vector field $\eta$ is unknown, as it is usual for actual processes, and the vector field $\varphi$ is uncertain such that $\varphi=\varphi_{o}+\Delta \varphi$ where $\varphi_{0}$ is some nominal function and $\Delta \varphi$ is the corresponding modeling error. Following this alternative representation of the system (7), it is possible to arrive to a representation that contains unknown and uncertain terms (11) or (12): 
Here,

$\zeta(\xi, \mu)=\dot{\eta}+\left(\frac{\partial \varphi_{o}}{\partial \xi} \frac{\partial \xi}{\partial t}+\frac{\partial \varphi_{o}}{\partial \mu} \frac{\partial \mu}{\partial t}+\Delta \dot{\varphi}+\dot{\varphi}_{0}\right) u+\Delta \dot{\varphi}$

is the whole uncertain term.

Now, proposing the following reaching law:

$\dot{s}=l_{1} s-l_{2} \operatorname{sign}(s)|s|^{1 / w}$

Here, $l_{1} \in[0,1)$ and $w \in Z^{+}$.

For controller design, let us consider the slidingmode surface and the proposed reaching law, from Equations 12 and 13, therefore, the following control law is obtained:

$\dot{u}=\varphi_{o}(\xi, \mu)^{-1}\left(l_{1} s-l_{2} \operatorname{sign}(s)|s|^{1 / w}-\zeta-\sum_{i=1}^{r+1} a_{i} \xi_{i}\right)$

Substituting the above expression onto Equation (12), the following closed-loop system is produced:

$\dot{\xi}_{i}=\xi_{i+1} ; i=1,2, \ldots, r-1$

$\dot{\xi}_{r}=\xi_{r+1}$

$\dot{\xi}_{r+1}=l_{1} s-l_{2} \operatorname{sign}(s)|s|^{1 / w}-\sum_{i=1}^{r+1} a_{i} \xi_{i}$

$\dot{\mu}=\gamma(\xi, \mu)$

$y=\xi_{1}$

Now, analyzing the closed-loop stability under ideal conditions, i.e. under the assumption of perfect model knowledge, let us propose the following Lyapunov function:

$V=\frac{s^{2}}{2}$
Considering its time derivative:

$\dot{V}=s\left(l_{1} s-l_{2} \operatorname{sign}(s)|s|^{1 / w} \leq \mid s\left(|s|-g_{2}|s|^{1 / w}\right)\right)<0$

The above, if

$l_{2}>|s|^{1-1 / w} \Rightarrow l_{2}>0$

\subsection{Non ideal case}

Let us consider a more real situation, where perfect model knowledge it is not available, i.e. the term $\zeta$ in (12) is unknown. Under the above, there are methodologies to generate the lack of model information, one of these is the so-called uncertainty estimators, which have been published in the literature, we consider an uncertainty estimator as proposed by (Aguilar, et. al. 2003), to infer the unknown term $\zeta$ and simultaneously filter the noise of the measured output. The estimation methodology is as follows:

$$
\begin{aligned}
& \dot{\xi}_{r+1}=\zeta(\xi, \mu)+\varphi_{o}(\xi, \mu) \dot{u} \\
& \dot{\xi}_{*}=\xi_{1}+\vartheta \\
& \dot{\varsigma}=\Theta(\xi, v) \\
& y_{*}=\xi_{*}
\end{aligned}
$$

The main issue of this new representation of the system is to eliminate the additive noise from the new system output $y_{*}$, transforming the original output disturbance into a system disturbance. So the uncertain term $\zeta$, is considered as a new state and $\Theta(\xi, v)$ is a nonlinear unknown function that describes its dynamics, which is assumed bounded. Here $\vartheta$ is an additive bounded measurement noise.

Proposition 1: The following dynamic system is an asymptotic-type observer of the system (19): 
$\hat{\xi}_{r+1}^{\bullet}=\hat{\zeta}+\hat{\varphi}_{o} \dot{u}+k_{1}\left(\xi_{*}-\hat{\xi}_{*}\right)$

$\dot{\xi}_{*}=\hat{\xi}_{1}+k_{2}\left(\xi_{*}-\hat{\xi}_{*}\right)$

$\dot{\varsigma}=k_{3}\left(\xi_{*}-\hat{\xi}_{*}\right)$

The vector of proportional observer gains,

$\mathbf{K}=\left(\begin{array}{lll}k_{1} & k_{2} & k_{3}\end{array}\right)$, is proposed as

$\mathbf{K}=-\mathbf{S}_{\theta}^{-1} \mathbf{C}^{T}$

$\mathbf{S}_{\theta}=\left(\frac{1}{\theta^{i+j-1}} S_{i j}\right)_{i, j=1, n+2}$

Parameter $\theta>0$ determines the desired convergence velocity. Moreover, in order to ensure stabilizing properties, $\mathbf{S}_{\theta}$ should be a positive solution of the algebraic Riccati equation:

$\mathbf{S}_{\theta}\left(\mathbf{E}+\frac{\theta}{2} \mathbf{I}\right)+\left(\mathbf{E}^{T}+\frac{\theta}{2} \mathbf{I}\right) \mathbf{S}_{\theta}=\mathbf{C}^{T} \mathbf{C}$

$\mathbf{E}=\left(\begin{array}{cc}0 & \mathbf{I}_{n-1, n-1} \\ 0 & 0\end{array}\right)$

The convergence properties of the above estimation methodology are given by Aguilar, et al. (Aguilar, et. al. 2003).

Now, employing the estimation procedure the non-ideal controller is expressed as

$$
\dot{u}=\hat{\varphi}_{o}^{-1}\left(l_{1} s-l_{2} \operatorname{sign}(s)|s|^{1 / w}-\hat{\zeta}-\sum_{i=1}^{r+1} a_{i} \hat{\xi}_{i}\right)
$$

This control law produces such practical closedloop stability that it can not cancel the nonlinearities completely, but it forces the trajectories to remain in a close neighborhood around of the set point or the reference trajectory.

\section{Mathematical Model of the Reactor}

The exothermic chemical reactor model considered here has been extensively studied (Jørgensen and Aris, 1983), it exhibits a complex or even chaotic dynamic behavior. In this system, temperature is regulated by manipulating the flow of water through a cooling jacket. A stream with a reactive $\gamma_{A}$ enters to the continuous reactor and is converted to an intermediate product $\gamma_{B}$, which reacts to transform into the final product $\gamma_{C}$; both reactions follow first order kinetics.

Concentrations are scaled with respect to initial concentration of reactive $\gamma_{A}\left(\gamma_{\text {Ao }}\right)$ (23 and 24) and characteristic times are scaled with respect to the mean residence time (25); temperature is scaled as it is shown by (26).

$$
\begin{gathered}
\alpha=\frac{\gamma_{A}}{\gamma_{\text {Ao }}} \\
\beta=\frac{\gamma_{B}}{\gamma_{\text {Ao }}} \\
\tau=\frac{t}{t_{\text {res }}}
\end{gathered}
$$

$\theta=\frac{E_{a}\left(T-T_{c}\right)}{R T_{c}^{2}}$

Introducing these dimensionless variables, a set of dimensionless mass and energy balance equations is obtained (27-29).

$$
\frac{d \alpha}{d \tau}=1-\alpha-\frac{1}{\tau_{c h}} \alpha \exp (\theta)
$$

$\frac{d \beta}{d \tau}=\frac{1}{\tau_{c h}} \alpha \exp (\theta)-\frac{1}{\tau_{c h}} \phi \beta \exp (\theta)-\beta$

$\frac{d \theta}{d \tau}=\frac{1}{\tau_{c h}} u \alpha \exp (\theta)+\frac{1}{\tau_{c h}} u \phi \beta \exp (\theta)-\left(1+\tau_{N}^{-1}\right) \theta$ 
In ordinary control notation, the vector of states can be written as $X^{T}=\left(\begin{array}{lll}\alpha & \beta & \theta\end{array}\right)$, being $\theta$ the only one regulated. Parameters related to the named chemical time, reaction rate ratio, dimensionless temperature of the cooling jacket and the Newtonian cooling time are, respectively,

$\tau_{c h}=\frac{1}{k_{1} t_{r e s}}$

$\phi=\frac{A_{2}}{A_{1}}$

$u=\theta_{j}=\frac{\left(-\Delta H_{1} a_{0} E_{a 1}\right)}{C p \rho R T_{c}^{2}}$

$\tau_{N}=\frac{t_{N}}{t_{\text {res }}}=\frac{C p \rho V}{U A_{c} t_{\text {res }}}$

An important characteristic of this reactor model is that it exhibits a minimum phase behavior, i.e., zero dynamics are stable when the temperature of the reactor is regulated.
Therefore, analysis of the inner dynamics concerns closed-loop behavior of $\gamma_{A}$ and $\gamma_{B}$ while $\theta$ is kept constant by the control. Therefore, the system (27-29) can be reduced to

${\stackrel{\bullet}{\gamma_{A}}}^{*}=1-\left(1+\varphi_{1}\right) \gamma_{A}^{*}$

$\dot{\gamma}_{B}^{*}=\delta_{1} \gamma_{A}^{*}-\left(1+\varphi_{2}\right) \gamma_{B}^{*}$

Here,

$\varphi_{1}=\frac{\exp \left(\theta_{s p}\right)}{\tau_{c h}}$

$\varphi_{2}=\frac{\phi \exp \left(\theta_{s p}\right)}{\tau_{c h}}$

Now, by solving the system (34) the following dynamic behavior is obtained:

$$
\begin{aligned}
& \gamma_{A}^{*}=\left[\gamma_{A o}^{*}-\left(\frac{1}{1+\varphi_{1}}\right)\right] \exp \left(-\left\{1+\varphi_{1}\right\} \tau\right)+\left(\frac{1}{1+\varphi_{1}}\right) \\
& \gamma_{B}^{*}=\left[\gamma_{B o}^{*}-\left(\varphi_{3}+\varphi_{4}\right)\right] \exp \left(-\left\{1+\varphi_{1}\right\} \tau\right)+\varphi_{3} \exp \left(-\left\{1+\varphi_{1}\right\} \tau\right)+\gamma_{4}
\end{aligned}
$$

Here,

$$
\begin{aligned}
& \varphi_{3}=\left(\frac{1}{\phi-1}\right)\left(x_{10}^{*}-\left[\frac{1}{1+\exp \left(\theta_{s p}\right) / \tau_{c h}}\right]\right) \\
& \varphi_{4}=\frac{\exp \left(\theta_{s p}\right) / \tau_{c h}}{\left(1+\exp \left(\theta_{s p}\right) / \tau_{c h}\right)\left(1+\phi \exp \left(\theta_{s p}\right) / \tau_{c h}\right)}
\end{aligned}
$$




\section{Dynamic Nonlinear Feedback for Temperature Control of Continuous Stirred Reactor with Complex Behavior, Pablo A. López Pérez, et, al, 202-217}

From the system (37), it is possible to note that inner dynamics of the reactor are asymptotically stable, i. e.

$\lim _{\tau \rightarrow \infty} \gamma_{A}^{*}=\frac{1}{\left(1+\exp \left(\theta_{s p}\right)\right) / \tau_{c h}}=\alpha_{e q}$

$\lim _{\tau \rightarrow \infty} \gamma_{B}^{*}=\frac{\exp \left(\theta_{s p}\right) / \tau_{c h}}{\left(1+\exp \left(\theta_{s p}\right) / \tau_{c h}\right)\left(1+\phi \exp \left(\theta_{s p}\right) / \tau_{c h}\right)}=\beta_{e q}$

Now, to provide a control notation, let us consider the following nonlinear dynamic system representation of the chemical reactor, considering an affine (linear) control law (42).

$$
\left[\begin{array}{c}
\dot{x_{1}} \\
\dot{x_{2}} \\
\dot{x_{3}}
\end{array}\right]=\left[\begin{array}{c}
\zeta_{1}(X) \\
\zeta_{2}(X) \\
\zeta_{3}(X)
\end{array}\right]+\left[\begin{array}{ccc}
g_{1} & 0 & 0 \\
0 & g_{2} & 0 \\
0 & 0 & g_{3}
\end{array}\right]\left[\begin{array}{l}
0 \\
0 \\
u
\end{array}\right]
$$

Here, vector function $\zeta(X)$ contains the nonlinear kinetic terms of the model, which can be expressed as function of the other system parameters (43), and vector $\ell$ entries are the coefficients of the control input (44-46).

$$
\begin{gathered}
\zeta(X)=\left[\begin{array}{l}
\zeta_{1}(X) \\
\zeta_{2}(X) \\
\zeta_{2}(X)
\end{array}\right]=\left[\begin{array}{c}
-\frac{1}{\tau_{c h}} \alpha \exp (\theta) \\
\frac{1}{\tau_{c h}} \alpha \exp (\theta)-\frac{1}{\tau_{c h}} \phi \beta \exp (\theta) \\
-\left(1+\tau_{N}^{-1}\right) \theta
\end{array}\right] \\
g(X)=\left[\begin{array}{ccc}
g_{1} & 0 & 0 \\
0 & g_{2} & 0 \\
0 & 0 & g_{3}
\end{array}\right]=\left[\begin{array}{ccc}
1-\alpha & 0 & 0 \\
0 & -\beta & 0 \\
0 & 0 & \left(\frac{1}{\tau_{c h}} \alpha \exp (\theta)+\frac{1}{\tau_{c h}} \phi \beta \exp (\theta)\right)
\end{array}\right]
\end{gathered}
$$

From (42), it is possible to find the theoretical value (Maya-Yescas and Aguilar, 2003) of the vector of manipulate variables (45).

$$
U=\left[\begin{array}{c}
0 \\
0 \\
\theta_{j}
\end{array}\right]
$$

Finally, the system can be written in ordinary control notation as (46-47).

$$
\begin{aligned}
& \dot{X}=\zeta(X)+g(X) U \\
& Y=h(X)=C X
\end{aligned}
$$




\section{Numerical Experiments}

Numerical simulations for the closed-loop system were performed in order to show the properties of the control scheme proposed. The set of parameters of the chemical reactor are chosen as in Jorgensen and Aris, (1983), and initial conditions of the differential equations (9-11) are $\left[x_{1}=0.45, x_{2}=0.1, x_{3}=0.9\right]$. For comparison purposes, an ideal $\mathrm{I} / \mathrm{O}$ linearizing control, a standard sliding-mode and high order slidingmode controllers are implemented too; all of them employing the same values of the control gains as the proposed methodology, $l_{1}=10$ and $l_{2}=5$. The former temperature set point is $\theta_{s p}=3$, furthermore, the set point is changed from 3 to 2.5 at 30 time units and changed again at 2.0 at 50 time units, the nominal value of the control input is $u_{0}=17.5$; the controller is tunedon at $t=15$, the order of the high order sliding- mode controller is considered as $w=3$. Figure 1 shows the open-loop behavior of the corresponding space portrait; note that the oscillatory behavior of the corresponding trajectories makes the open-loop mode inadequate for industrial operation.

Closed loop performance of temperature trajectories show that the ideal I/O linearizing controller shows the better performance, so that it cancel the nonlinearities, imposing a desired linear behavior, with a satisfactory performance. The proposed controller tries to compensate the nonlinear terms, besides it is able to reach the set point value required (Figure 2 ), exhibiting smaller oscillations around the regulated trajectory than the other sliding-mode controllers. As predicted by the theoretical frame presented; sliding-mode and high order sliding-mode controllers can suppress nonlinear oscillations; however both controllers exhibit a considerable off-set from the corresponding set point.

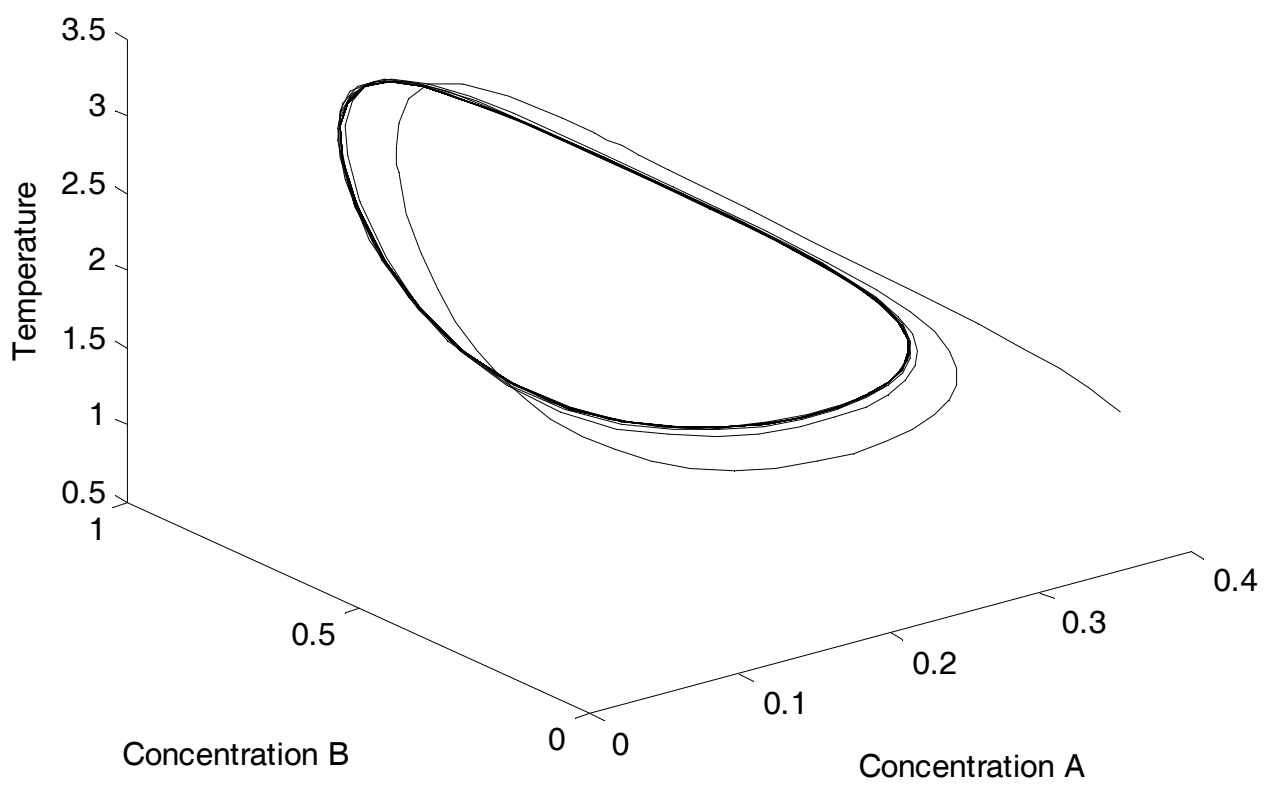

Figure 1. Phase portrait of open-loop behavior. 


\section{Dynamic Nonlinear Feedback for Temperature Control of Continuous Stirred Reactor with Complex Behavior, Pablo A. López Pérez, et, al, 202-217}

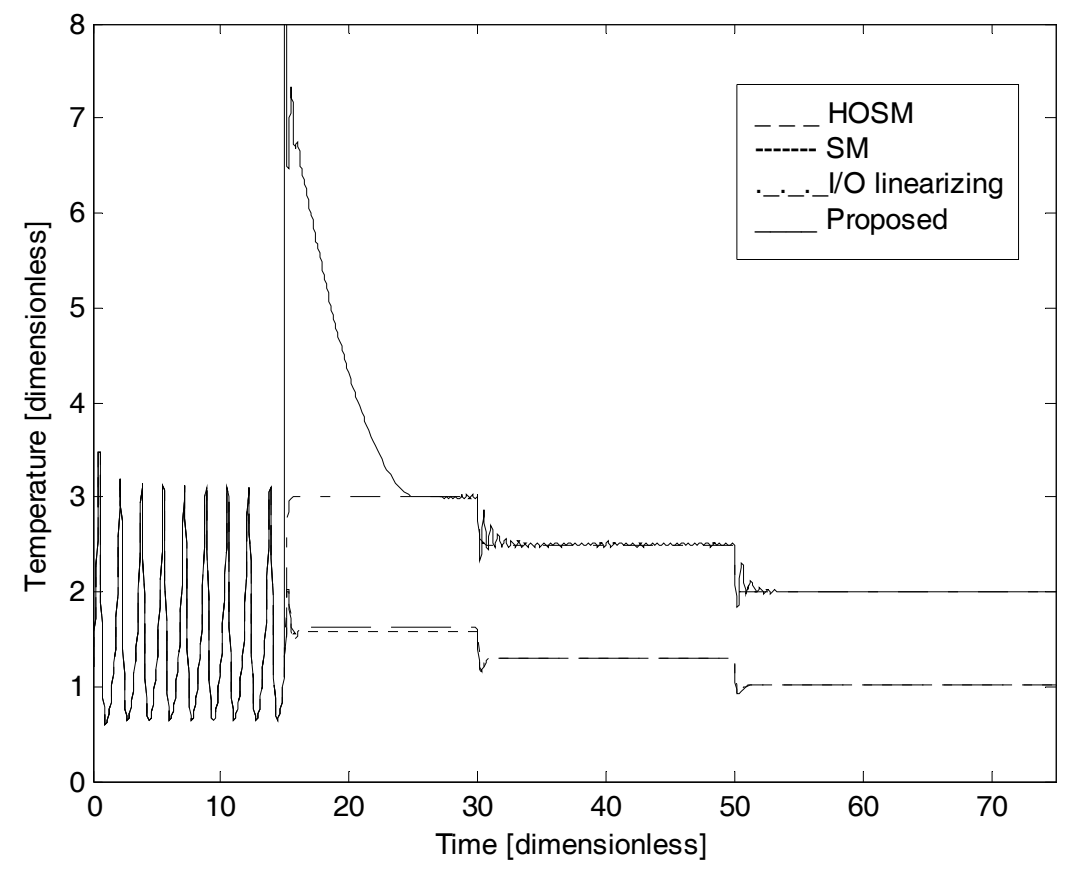

Figure 2. Closed-loop temperature trajectories with different control inputs.

Figure 3 is related to the uncontrolled state variables (reactive and product concentrations). In this figure, the corresponding oscillatory behavior is shown during the open-loop operation. When the proposed controller is turned on, this state variable reaches an stable steady state; this is in accordance with the minimum phase characteristic of this chemical reactor as shown above.

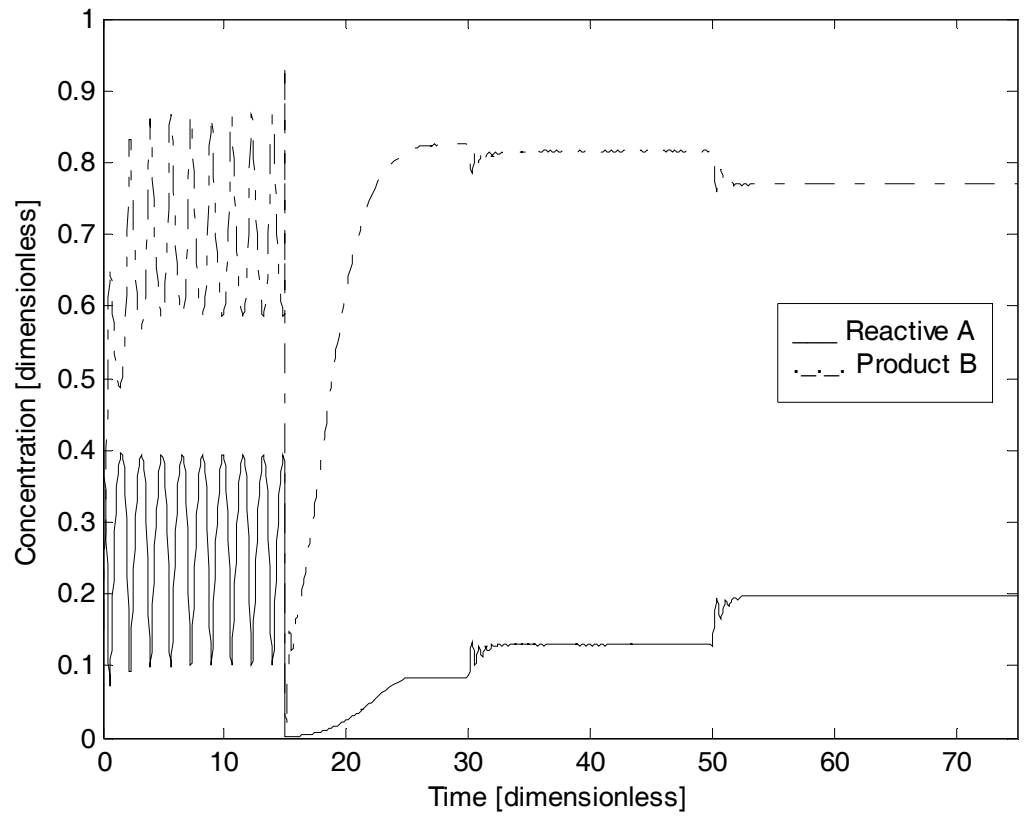

Figure 3. Closed-loop behavior of the uncontrolled states considering the proposed controller. 
Another important difference is that the effort performed by the manipulate variable (Figure 4) is very different for each controller. As can be noticed, the 1/O linearizing controller posses the best performance, the sliding-mode controller exhibits the second smoothest behavior, followed by the high-order sliding-mode control, which exhibits a more demanding effort at the start up of the regulation task. Finally, the proposed methodology presents the more demanding control action, where small oscillations are present.

By comparing the performance and control effort, it is possible to note that the high-order slidingmode control is not very efficient because the regulated variable, temperature, exhibits the largest off-set, even when the effort is higher than in the case of the sliding-mode controller; nevertheless, the performance of the slidingmode controller is not satisfactory because the set point is not reached. At the expense of a higher control effort, the I/O linearizing controller and the proposed controller are able to reach the set point; this is not a disadvantage because this controller is able to force the temperature trajectory through the desired value. The tuning of the sliding-mode feedback injections is complicated and, at present, guidelines for the tuning of this type of controllers are still under development. From the above, if the closed-loop trajectories of the system remain in a close neighborhood of the sliding surface, some instability would appear. For the proposed study case, the value of the control gains, $l_{1}$ and $l_{2}$, has to be chosen very carefully; other numerical simulations (not presented here) showed that, sometimes (smaller values of control gains), it is not possible to stabilize the oscillatory behavior of the chemical reactor whereas for large values of these parameters, it is possible to lead to unacceptable control efforts, offset or, even worse, to provoke additional closed-loop instabilities.

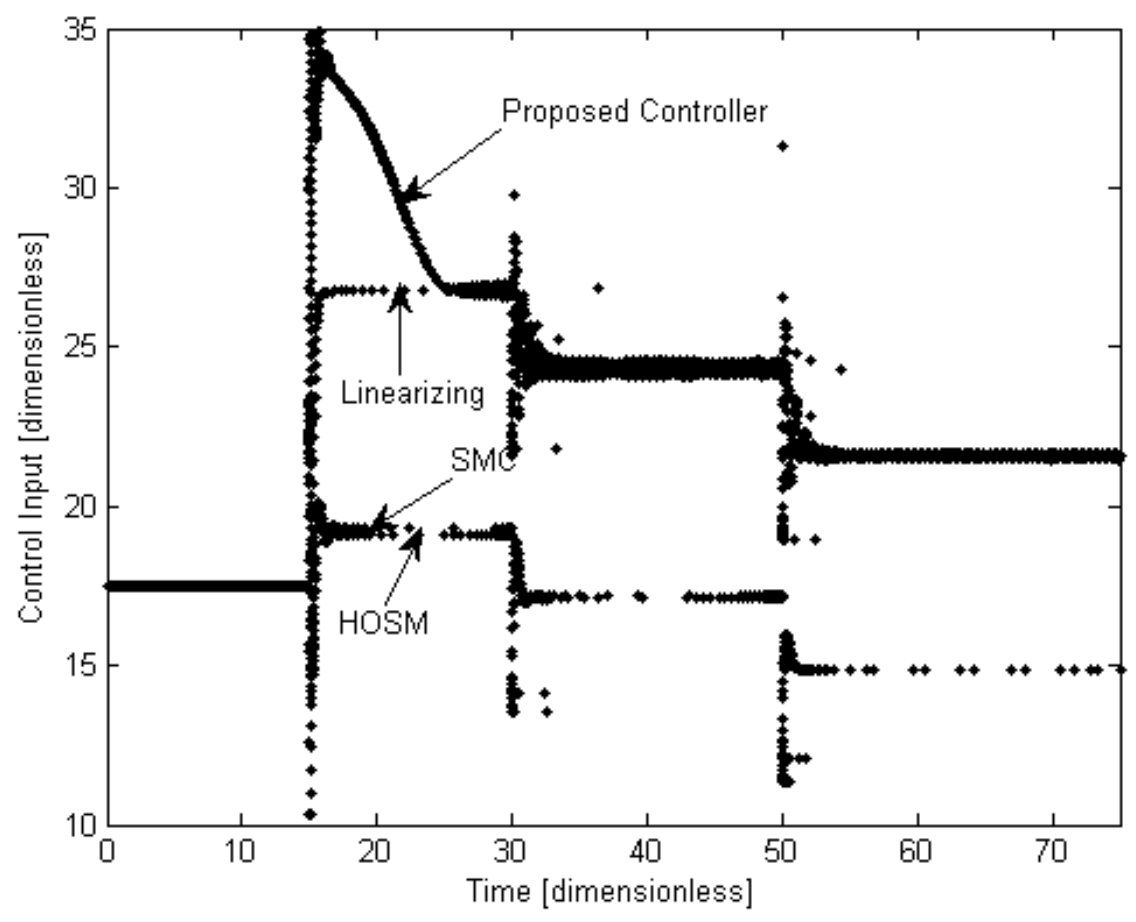

Figure 4. Control efforts. 


\section{Dynamic Nonlinear Feedback for Temperature Control of Continuous Stirred Reactor with Complex Behavior, Pablo A. López Pérez, et, al, 202-217}

In order to quantify the performance of the compared controllers, what Ogunnaike and Ray suggested is taken into account, that is, the Integral Time-Weighted Squared Error (ITSE) defined by (48). ITSE exhibits the advantage of heavy penalization of large errors at long time; therefore, it is a good measure of resilience of the controller.

$$
\text { ITSE }=\int_{0}^{\infty} t \varepsilon^{2} d t
$$

In order to compare the resilience of the controllers simulated, the ITSE was evaluated for the dynamic system under the influence of the three controllers (Figure 5). As it is possible to note, and confirming the findings from Figures 2 and 4 , the $1 / 0$ linearizing and the proposed controller are the only two able to stabilize the system in the long time whereas for the sliding- mode and high order sliding- mode controllers, this error increases unlimitedly. This result is due to the ability of the controller proposed to eliminate offset, property which is not exhibited by the other two controllers (Figure 2).

\section{Concluding remarks}

This work presents a class of integral high order sliding-mode controllers under the frame of differential algebra in order to transform the system to a canonical controllable form; where the internal dynamic is stable, an estimator is coupled to the proposed controller to infer the on measured and uncertain terms. The proposed controller is able to control the corresponding reactor temperature trajectories successfully in comparison with other nonlinear controllers, dismissing the chattering of the standard slidingmode controllers.

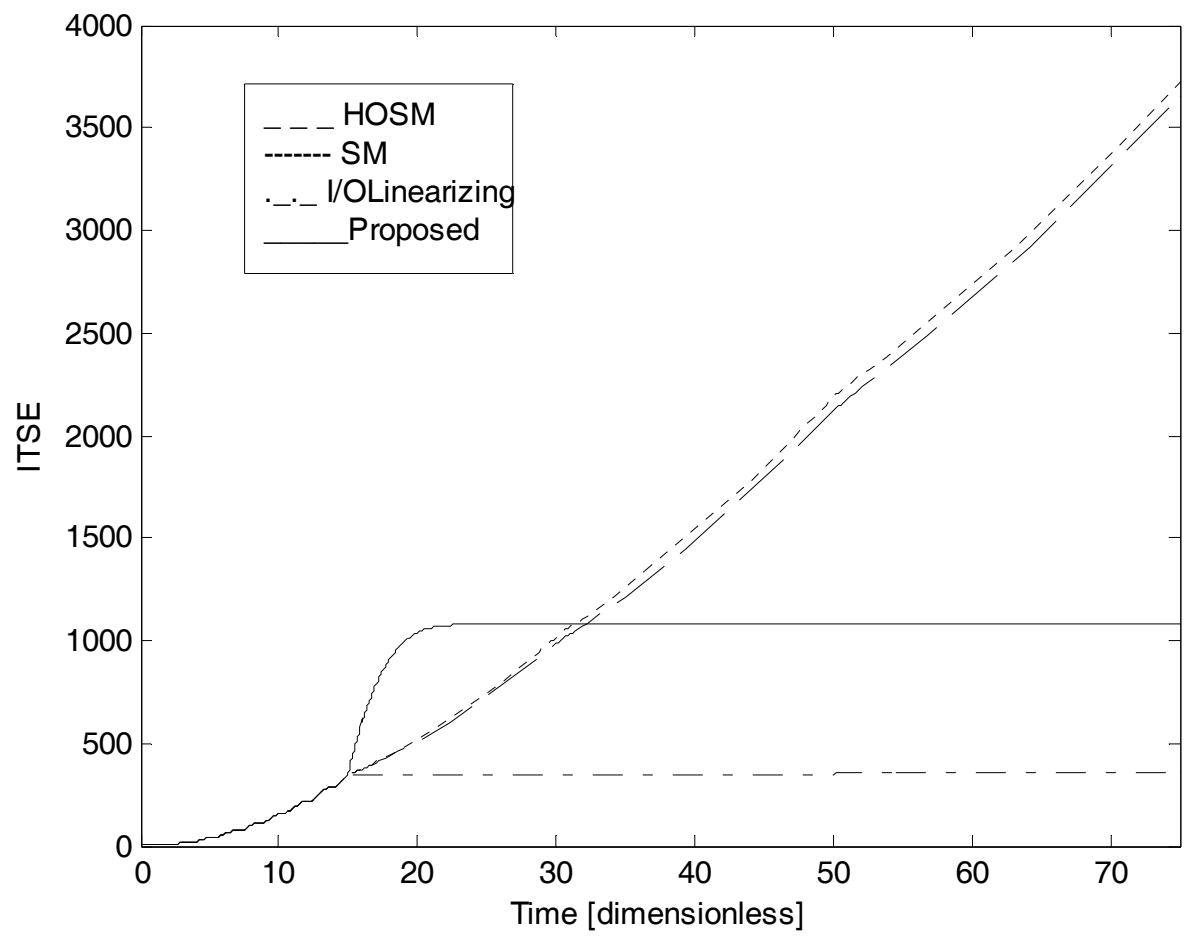

Figure 5. Performance Index. 


\section{Nomenclature}

a. - concentration of reactive $A$

Ac .- heat transfer area

$b$.- concentration of reactive $B$

$C p$.- heat capacity at constant pressure

$t$.- chronologic time

$T$.- temperature

$U$.- global heat transfer coefficient

$u$.- control input

$\boldsymbol{x}$.- vector of state variables

$Y$.- measured output

Greek letters.

$\zeta .-$ nonlinear vector

$\alpha$.- dimensionless concentration of reactive $A$

$B$.- dimensionless concentration of reactive $B$

$\rho$.- density

$\vartheta$.- dimensionless temperature

$\varepsilon$.- control error

$\Delta$.- nonlinear bound

$\Delta H j$.- heat of $\mathrm{j}$-th reaction

$\tau$.- dimensionless time

\section{References}

[1] Aris, R. and Admundson, N. An analysis of chemical reactor stability and control. Chem. Engng Sci. Vol. 7, 1958, pp. 121-155.

[2] Ricardo Aguilar, R. Martínez-Guerra and Rafael Maya-Yescas. State Estimation for Partially Unknown Nonlinear Systems: A Class of Integral High Gain Observers. IEE Proceedings Control Theory and Applications. Vol. 150, No.3, 2003, pp. 240-244.

[3] Ricardo Aguilar-López and Rafael Martínez-Guerra. Control of chaotic oscillators via a class of model free active controller: suppression and synchronization. Chaos, Solitons and Fractals.Vol. 38, , 2008, pp. 531-540.

[4] Ricardo Aguilar, Jesús González, José Alvarez and Miguel Angel Barrón. Temperature regulation of a class of continuous chemical reactor based on a nonlinear Luenberger-like observer. Journal of Chemical Technology and Biotechnology. Vol. 70, No. 3, 1997, pp. 209-216.
[5] Samuel Bowong, FM Moukam, Kakmeni and Clement Tchawoua. Controlled synchronization of chaotic systems with uncertainties via a sliding mode control design. Physical Review E. Vol. 70, No. 066217, 2004, pp. 1-8,

[6] Oscar Camacho and Carlos A. Smith. Sliding mode control: an approach to regulate nonlinear chemical processes. ISA Transactions. Vol. 39, No. 2, April 2000, pp. 205-218.

[7] C.Edwards and S.Spurgeon, Sliding Mode Control. Taylor and Francis, 1998.

[8] Girin, A.; Plestan, F.; Brim, X.; Glumineau, A. "A 3rd order sliding mode controller based on integral sliding mode for an electropneumatic system," 2006 45th IEEE Conference on Decision and Control, Vol. 45, No. 4, 1315 Dec. 2006, pp. $1617-1622$

[9] J. González, R. Aguilar, J. Alvarez-Ramirez and M. A. Barrón. Linearizing control of a binary distillation column based on a neuro-estimator. Artificial Intelligence in Engineering. 1999. Vol. 13, pp. 405-412.

[10] Isidori, A. Nonlinear Control Theory. 3d Edition. Springer-Verlag, 1995).

[11] Jorgensen, D.V. \& Aris, R. On the dynamics of a stirred tank with consecutive reactions. Chemical Engineering Science. Vol. 38, 1983, pp. 45-53.

[12] Kolavennu, S. Palanki, S. and Cockburn, J. Robust control of I/O linealizable systems via multi-model $\mathrm{H}_{2} / \mathrm{H} \infty$ Synthesis. Chem. Eng. Sci. Vol. 55, 2000, pp. 1583-1589.

[13] Salah Laghrouche, Franck Plestan, Alain Glumineau, Higher order sliding mode control based on integral sliding mode, Automatica, Vol. 43, No. 3, March 2007, pp. 531-537.

[14] Levant, A. Universal single-input-single-output (SISO) sliding-mode controllers with finite-time convergence. IEEE Transactions on Automatic Control. Vol. 46, No. 9, 2001, pp. 1447-1451.

[15] Levant, A. Sliding order and sliding accuracy in sliding mode control. Int. J. of Control. Vol. 58, 1993, pp. 1247-1263. 
[16] R. Maya-Yescas and R. Aguilar. Controllability assessment approach for chemical reactors: non-linear control affine systems. Chemical Engineering Journal.. Vol. 92, No. 1, 2003, pp. 69-79.

[17] Janos Madar, Janos Abonyi and Ferenc Szeifert. Feedback linearizing control using hybrid neural networks identified by sensitivity approach. Engineering Applications of Artificial Intelligence. Vol. 18, No. 3, April 2005, pp. 343-351.

[18] Mohamed Azlan Hussain. Review of the applications of neural networks in chemical process control - simulation and online implementation. Artificial Intelligence in Engineering. Vol. 13, No. 1, January 1999, pp. 55-68.

[19] M.I. Neria.González and R. Aguilar-López. Tracking Trajectories in a Continuous Anaerobic Bioreactor employing a Nonlinear Proportional Controller. Int. Journal of Chemical Reactor Engineering. Vol. 5, A73, 2007.

[20] M.I. Neria.González, R. Martínez-Guerra and R. Aguilar-López. Feedback regulation of an industrial aerobic wastewater plant. Chemical Engineering Journal. Vol. 139, 2008, pp. 475-481.

[21] Ogunnaike, B.A. and Ray, W.H. Process, Dynamics, Modeling and Control. Oxford University Press. New York, 1994.

[22] F.G. Shinskey. Feedback Controllers for the Process Industries. Mc Graw Hill. 1994

[23] V.Utkin, J.Guldner, and J.Shi. Sliding Modes in Electromechanical Systems. Taylor and Francis, 1999.

\section{Acknowledgement}

PALP is grateful with CONACYT for the corresponding postgraduate scholarship 


\section{Authors Biography}

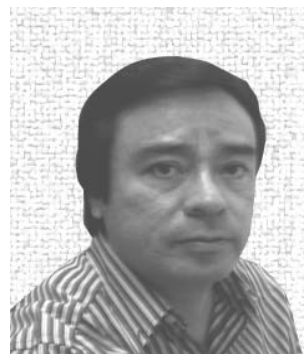

\section{Ricardo Aguilar López}

He was born in Mexico City in 1964. He earns his B.Sc., M. Sc. and Ph.D. degrees from Universidad Autónoma Metropolitana in 1989, 1993 and 1998, respectively, all of them in chemical engineering. He was a postdoctoral fellow at Instituto Mexicano del Petróleo (2000); besides, he earns a Ph.D. in automatic control from CINVESTAV-IPN (2003). He works in robust observer design for nonlinear systems and process control. He is author and co-author of 83 papers published in international journals. Currently, he is a researcher at the Biotechnology and Bioengineering Department of the Centro de Investigación y Estudios Avanzados of IPN and member of the National System of Researchers since 1998 (level II, currently).

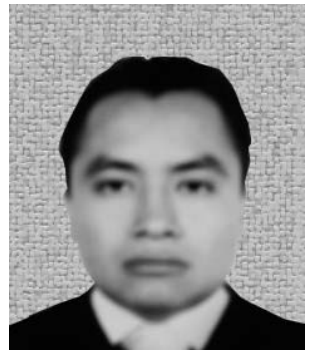

\section{Pablo Antonio López Pérez}

He was born in Mexico City. He earns a B.Sc. in chemical engineering from the Universidad Autónoma Metropolitana. He is a graduate student in biotechnology and bioengineering from CINVESTAV IPN. Mr. Lopez has worked in automatic control of processes, linear and nonlinear control and instrumentation and monitoring. His main interest is in simulation and control of industrial bioprocesses. 